Elect. Comm. in Probab. 16 (2011), 405-422

ELECTRONIC

COMMUNICATIONS

in PROBABILITY

\title{
HALF INDEPENDENCE AND HALF CUMULANTS
}

\author{
ARUP BOSE $^{1}$ \\ Statistics and Mathematics Unit, Indian Statistical Institute, 203 B.T. Road, Kolkata 700108. \\ email: bosearu@gmail.com
}

RAJAT SUBHRA HAZRA

Statistics and Mathematics Unit, Indian Statistical Institute, 203 B.T. Road, Kolkata 700108.

email: rajat_r@isical.ac.in

KOUSHIK SAHA

Bidhannagar Government College, Sector I, Salt Lake, Kolkata 700064.

email: koushiksaha877@gmail.com

Submitted November 2, 2010, accepted in final form April 5,2011

AMS 2000 Subject classification: Primary 60B20; Secondary 46L53, 46L54, 60B10

Keywords: Cramer's theorem, Central limit theorem, cumulant, $C^{*}$ probability space, free algebras, free independence, half commutativity, half independence, noncommutative probability spaces, reverse circulant matrix, Rayleigh distribution, semicircular law, symmetric partition.

\section{Abstract}

The notion of half independence arises in random matrices and quantum groups. This notion is available only for elements of a noncommutative probability space and assumes the existence of all moments. We relate half independence to a certain class of partitions and use it to define an appropriate cumulant generating function and a transform which is closely related to the characteristic function. This leads to a definition of half independent convolution of arbitrary probability measures which is compatible with the distribution of the sum of half independent elements of a noncommutative probability space. We also establish the central limit theorem for half independent convolution of measures with the limit being symmetrized Rayleigh. Cramer's theorem is also established in this set up.

\section{Introduction}

Along with classical independence, another well known notion of independence is free independence in the noncommutative set up. A third notion of independence in the noncommutative set up is half independence. This has been described in Banica, Curran and Speicher (2010) [2] and Bose, Hazra and Saha (2010)[7]. In Section 2, we provide a quick description of these three notions and two examples of half independence. The goal of this article is to study half independence in details.

\footnotetext{
${ }^{1}$ RESEARCH SUPPORTED BY J.C.BOSE FELLOWSHIP, GOVERNMENT OF INDIA. PART OF THE WORK DONE WHILE VISITING DEPARTMENT OF ECONOMICS, UNIVERSITY OF CINCINNATI IN OCTOBER 2010.
} 
To motivate our results, let us recall the results from classical and free independence that are relevant to us. In classical independence, the two natural transforms, which are measure determining and convergence determining, are the characteristic function $\phi(\cdot)$ and the cumulant generating function $\chi(\cdot)$. For any probability measure $\mu$ on $\mathbb{R}$, these are defined as

$$
\phi_{\mu}(t)=\int_{\mathbb{R}} e^{i t x} d \mu(x) \text { and } \chi_{\mu}(t)=\log \phi_{\mu}(t) \text { (for } t \text { in a neighbourhood of } 0 \text { ). }
$$

If $\mu$ and $v$ are two probability measures and $\mu * v$ is their independent (additive) convolution, then

$$
\chi_{\mu * v}(t)=\chi_{\mu}(t)+\chi_{v}(t) .
$$

Well known results for this convolution are the CLT and Cramer's theorem.

Initially, free independence of noncommutative subalgebras or elements was defined via relations between moments. It is well known that noncrossing partitions play a crucial role in free independence. Later Cauchy transform and the R-transform (or Voiculescu transform) (see Nica and Speicher (2006) [10], Anderson et al. (2009)[1]) were defined. This helped to define free convolution of arbitrary probability measures. For instance the $R$-transform satisfies equation (1) when classical convolution is replaced by the free convolution. The free CLT holds: the $n$ fold free convolution of identical laws with finite second moment, when scaled by $\sqrt{n}$ and centered, converges to the semicircle law. See Nica and Speicher (2006)[10]. However, Cramer's theorem fails: free convolution of two laws may be semicircular without the individual laws being semicircular. See Bercovici and Voiculescu (1995)[5].

The existing notion of half independence makes sense only for elements of a noncommutative probability space via the behavior of their moments. It is known that this definition does not extend to half independence between subalgebras (see Speicher (1997)[11]) and no notion of half independence for arbitrary random variables is available in the literature.

The above article as well as the work of Bose, Hazra and Saha (2010)[7] suggests that symmetric partitions play a significant role in half independence. In Section 3 we use symmetric partitions to develop notions of half cumulants and half cumulant generating function under suitable restrictions on the growth of the moments. Then we relate the moments to the half cumulants via appropriate generating functions. This relationship helps us to go beyond the set up of noncommutative algebra and provides an analytical definition of half independent convolution of probability measures. First, in Section 4 we develop the notion of an appropriate transform $T_{\mu}(\cdot)$ for any symmetric (about zero) probability measure $\mu$. This transform plays the same role in half independence as do the characteristic function in classical independence and Cauchy transform in free independence. This transform $T$ has a surprisingly simple description in terms of the characteristic function and is similar to the radial characteristic function defined in Kingman (1963)[8]. Let $\circledast$ denote the product convolution. Then it turns out that

$$
T_{\mu}(t)=\phi_{\mu \circledast \rho}(2 t) \text { for all } t \in \mathbb{R}
$$

where $\rho$ is the arcsine law with density

$$
\frac{2}{\pi} \frac{1}{\sqrt{1-\alpha^{2}}}, 0<\alpha<1
$$

$T$ is both measure determining and convergence determining (see Lemma 2 below). We define the half cumulant generating function as,

$$
H(t)=\log T(t) \text { (in an appropriate neighbourhood of } 0 \text { ). }
$$


The half independent convolution $\mu \oslash v$, of symmetric probability measures $\mu$ and $v$ is defined as the (unique) probability measure which satisfies

$$
T_{\mu \odot v}(t)=T_{\mu}(t) T_{v}(t) \text { for all } t \in \mathbb{R} .
$$

and hence the corresponding half cumulant generating functions satisfy

$$
H_{\mu \oslash v}(t)=H_{\mu}(t)+H_{v}(t) \text { (for } t \text { in a neighbourhood of } 0 \text { ). }
$$

This half convolution is quite similar to the convolution defined by Kingman (1963) [8] who arrived at it in a completely different context.

This definition of convolution is compatible with addition of half independent variables in a noncommutative probability space: if $a$ and $b$ are half independent in some $C^{*}$-probability space with laws $\mu$ and $v$, then the law of $(a+b)$ exists and is given by $\mu \oslash v$.

In half independence Rayleigh law takes the place of Gaussian law in independence and the semicircular law in free independence. The symmetrized Rayleigh law $R_{\sigma}$ has the density

$$
f(x)=\frac{|x|}{\sigma^{2}} \exp \left(-x^{2} / \sigma^{2}\right),-\infty<x<\infty
$$

with moments

$$
\beta_{2 k+1}=0 \text { and } \beta_{2 k}=\sigma^{2 k} k \text { ! for all } k \geq 0 .
$$

If $\sigma=1$, then it is known as standard symmetrized Rayleigh law and is denoted by $R$.

In Section 5, we establish Cramer's theorem: $\mu \oslash v$ is symmetrized Rayleigh, if and only if both $\mu$ and $v$ are symmetrized Rayleigh. We also prove the half independent CLT: the $n$ fold half independent convolution of symmetric laws satisfying suitable moment condition, when scaled by $\sqrt{n}$, converges to the symmetrized Rayleigh distribution. An appropriate CLT in a $C^{*}$-algebra also holds and is proved by computation of moments using symmetric partitions.

\section{The three notions of independence}

\subsection{Preliminaries of noncommutative probability spaces}

Let $(\mathscr{A}, \tau)$ be a noncommutative $*$-probability space where $\mathscr{A}$ is a $*-$ unital complex algebra (with unity 1 ) and $\tau: \mathscr{A} \rightarrow \mathbb{C}$ is a linear functional satisfying $\tau(1)=1$. At times we shall assume $\tau$ is a state, that is $\tau(a) \geq 0$ if $a \geq 0$ ( $a \geq 0$ means $a^{*}=a$ and its spectrum $\operatorname{sp}(a)$ is nonnegative). The elements of the algebra will be referred to as random variables and we shall concentrate only on self adjoint random variables. By the law of self adjoint $a \in \mathscr{A}$, we mean the collection $\left\{m_{k}(a)\right\}$ where

$$
m_{k}(a)=\tau\left(a^{k}\right), k \geq 1
$$

are the moments of $a$. Suppose $\tau$ is a state and $a$ is a self adjoint random variable. Then given any $n$ and any collection of complex numbers $\left\{c_{i}, 0 \leq i \leq n\right\}$

$$
\sum_{k=0}^{n} \sum_{l=0}^{n} c_{k} \tau\left(a^{k+l}\right) \overline{c_{l}}=\tau\left(\left(\sum_{k=0}^{n} c_{k} a^{k}\right)\left(\sum_{l=0}^{n} c_{l} a^{l}\right)^{*}\right) \geq 0 .
$$

Then $\left\{m_{k}(a), k \geq 0\right\}$ is a moment sequence, and there exists a measure $\mu_{a}$ on the real line such that

$$
m_{k}(a)=\tau\left(a^{k}\right)=\int x^{k} d \mu_{a}(x)
$$


$\mu_{a}$ may not be unique. A sufficient condition for $\mu_{a}$ to be unique is Carleman's condition

$$
\sum\left[\tau\left(a^{2 k}\right)\right]^{-1 /(2 k)}=\infty .
$$

In that case we also call $\mu_{a}$ to be the law of $a$.

When $(\mathscr{A}, \tau)$ is a $C^{*}$-algebra (that is, $\mathscr{A}$ is a $*$-algebra equipped with a norm $\|\cdot\|$ such that $\|a b\| \leq\|a\|\|b\|$ and $\left\|a a^{*}\right\|=\|a\|^{2}$ for $a, b \in \mathscr{A}$ ), the law $\mu_{a}$ of $a$ exists and is compactly supported. More generally, suppose that the algebra is not necessarily $C^{*}$ and $\tau$ is a state and for the self adjoint random variable $a, \tau\left(a^{2 k}\right) \leq C^{k} k$ ! for all $k$. Then $\left\{\tau\left(a^{2 k}\right)\right\}$ satisfies condition (4), and hence $\mu_{a}$ is the law of $a$ but it is not necessarily compactly supported.

For random variables $\left\{a_{i}\right\}_{i \in J}$, their joint moments are the collection $\left\{\tau\left(a_{i_{1}} a_{i_{2}} \ldots a_{i_{k}}\right), k \geq 1\right\}$, where each $a_{i_{j}} \in\left\{a_{i}\right\}_{i \in J}$.

Random variables $\left\{a_{i, n}\right\}_{i \in J} \in\left(\mathscr{A}_{n}, \tau_{n}\right)$ are said to converge in law to $\left\{a_{i}\right\}_{i \in J} \in\left(\mathscr{A}^{\prime}, \tau^{\prime}\right)$ if each joint moment of $\left\{a_{i, n}\right\}_{i \in J}$ converges to the corresponding joint moment of $\left\{a_{i}\right\}_{i \in J}$.

\subsection{Independence: free, classical, and half}

Unital subalgebras $\left\{\mathscr{A}_{i}\right\}_{i \in J} \subset \mathscr{A}$ are called freely independent or simply free if

$$
\tau\left(a_{j}\right)=0, a_{j} \in \mathscr{A}_{i_{j}} \text { and } i_{j} \neq i_{j+1} \text { for all } j \Rightarrow \tau\left(a_{1} \cdots a_{n}\right)=0 .
$$

Random variables (or elements of an algebra) $\left\{a_{1}, a_{2}, \cdots\right\}$ are called free if the subalgebras generated by them are free.

Unital subalgebras $\left\{\mathscr{A}_{j}\right\}_{j \in J} \subset \mathscr{A}$ are said to be independent if they commute and

$$
\tau\left(a_{1} \cdots a_{n}\right)=\tau\left(a_{1}\right) \cdots \tau\left(a_{n}\right) \text { for all } a_{j} \in \mathscr{A}_{i_{j}} \text { where } k \neq l \Rightarrow i_{k} \neq i_{l} .
$$

Two elements $a$ and $b$ of any algebra are said to be independent if the two unital algebras generated by them are independent.

Let $\left\{a_{i}\right\}_{i \in J}$ be noncommutative elements of $(\mathscr{A}, \tau)$. We say that they half commute if

$$
a_{i} a_{j} a_{k}=a_{k} a_{j} a_{i}
$$

for all $i, j, k \in J$. Clearly, if $\left\{a_{i}\right\}_{i \in J}$ half commute then $a_{i}^{2}$ commutes with $a_{j}$ and $a_{j}^{2}$ for all $i, j \in J$. The random variable $a=a_{i_{1}} a_{i_{2}} \ldots a_{i_{n}}$ where each $a_{i_{j}} \in\left\{a_{i}\right\}_{i \in J}$, is said to be balanced (with respect to $\left\{a_{i}\right\}$ ), if each random variable $a_{i}$ appears same number of times in odd and even positions of $a$. If $a$ is not balanced, we say it is unbalanced. So if $n$ is odd then $a$ is automatically unbalanced.

Definition 1. Half commuting elements $\left\{a_{i}\right\}_{i \in J}$ are said to be half independent if the following hold:

1. The variables $\left\{a_{i}^{2}\right\}_{i \in J}$ are independent.

2. If $a=a_{i_{1}} a_{i_{2}} \cdots a_{i_{n}}$ is unbalanced with respect to $\left\{a_{i}\right\}_{i \in J}$, then $\tau(a)=0$.

This definition of half independent elements is equivalent to that given in Banica, Curran and Speicher (2009)[2]. Note that, from the second condition the odd moments of half independent elements are zero.

The three notions of independence are different from each other. See Bose, Hazra and Saha (2010)[7] for details. Here are two examples of half independence. 
Example 1. (Banica, Curran and Speicher (2009) [2]) Let $(\Omega, \mathscr{B}, \mu)$ be a probability space and let $\left\{\psi_{i}\right\}$ be a family of independent complex Gaussian random variables. Define $a_{i} \in\left(M_{2}(L(\mu)), \mathrm{E}[\operatorname{tr}(\cdot)]\right)$ by

$$
a_{i}=\left[\begin{array}{cc}
0 & \psi_{i} \\
\psi_{i} & 0
\end{array}\right]
$$

where $\operatorname{tr}(\cdot)$ is normalized trace and $\overline{\psi_{i}}$ is the complex conjugate of $\psi_{i}$. Then $\left\{a_{i}\right\}$ are half independent. The law of each $a_{i}$ is a symmetrized Rayleigh distribution.

Example 2. (Bose, Hazra and Saha (2010)[7]) A reverse circulant matrix is an $n \times n$ symmetric matrix whose $(i, j)$-th entry is given by $x_{(i+j)} \bmod n$. The sequence $\left\{x_{i}\right\}$ is called the input sequence. Let $\left\{R C_{i, n}\right\}_{1 \leq i \leq p}$ be an independent (across $i$ ) sequence of $n \times n$ reverse circulant matrices each with an independent input sequence with mean 0 , variance 1 and for all $k \geq 1$,

$$
\sup _{n \in \mathbb{N}} \sup _{1 \leq i \leq p} \sup _{1 \leq m \leq l \leq n} \mathrm{E}\left[\left|R C_{i, n}(m, l)\right|^{k}\right] \leq c_{k}<\infty .
$$

Then $\left\{n^{-1 / 2} R C_{i, n}\right\}_{1 \leq i \leq p} \in\left(M_{n}(L(\mu)), \mathrm{E}[\operatorname{tr}(\cdot)]\right)$, converges to half independent $\left\{a_{1}, a_{2}, \ldots, a_{p}\right\}$ where $a_{i}$ is as in Example 1 with $\mathrm{E}\left|\psi_{i}\right|^{2}=1$.

Incidentally, in Proposition 2.8 of Banica, Curran and Speicher (2010)[2] it was shown that if $\left\{x_{i}\right\}$ are half independent elements in a $C^{*}$ probability space, then there exists independent complex valued random variables $\left\{\psi_{i}\right\}$ with $\mathrm{E}\left[\psi_{i}^{n} \overline{\psi^{m}}\right]=0$ for $m \neq n$ and $x_{i}$ has the same distribution as $y_{i}$ where

$$
y_{i}=\left[\begin{array}{cc}
0 & \psi_{i} \\
\psi_{i} & 0
\end{array}\right] .
$$

The above result reduces the study of half independence to the study of $2 \times 2$ matrices. However, we emphasize that this is true only in a $C^{*}$ probability space. There is no existing notion of half independence of arbitrary random variables or probability measures.

Remark 1. As pointed out above, there is no existing notion of half independence for random variables affiliated to some von Neumann algebra. One may proceed along the lines of Bercovici and Voilculescu (1993) [4] to define half independence for self adjoint affiliated random variables. Given two symmetric measures $\mu$ and $v$, it would be interesting to get hold of two self adjoint affiliated random variables which are half independent and have laws $\mu$ and $v$. We provide some lead to this approach in Subsection 4.2.

In particular, given a sequence of measures with unbounded support, one does not know how to find half independent random variables with these distributions. We shall address this issue in Section 4.

\section{Symmetric partitions and half independence}

The proof of the above matricial limit (Example (2)) given in Bose, Hazra and Saha (2010) [7] suggests that there is a suitable class of partitions which is tied to the notion of half independence just as noncrossing partitions are tied to free independence. In this section, we show that symmetric partitions may be used to develop a suitable notion of half cumulants. The corresponding half 
cumulant generating function captures the notion of half independence. This will serve as the precursor of the general notion of half cumulants and half cumulant generating function for arbitrary symmetric probability measures to be developed in the next section.

Any set $K$ of integers will be called symmetric if it has an equal number of odd and even integers. Any partition $\pi$ of $K$ will be called symmetric if each partition block is symmetric. The set of all symmetric partitions of $\{1,2, \ldots, 2 n\}$ will be denoted by $E(2 n)$. Given a sequence $\left\{l_{n}: \mathscr{A}^{n} \rightarrow \mathbb{C}\right\}$ of maps and partition $\pi$ of $\{1,2, \ldots, n\}$ define

$$
l_{\pi}\left(a_{1}, a_{2}, \ldots, a_{n}\right)=\prod_{V \in \pi} l_{|V|}\left(a_{j_{1}}, \ldots, a_{j_{|V|}}\right),
$$

where $V=\left(j_{1}, j_{2}, \cdots, j_{|V|}\right)$ is a block of the partition $\pi$.

Definition 2. Let $(\mathscr{A}, \tau)$ be a noncommutative probability space. Let $\left\{a_{i}\right\}_{i \in J}$ be random variables in $\mathscr{A}$ and suppose that $\tau\left(a_{i_{1}} \cdots a_{i_{k}}\right)=0$ when $k$ is odd and $i_{i}, \cdots, i_{k} \in J$. Then define half cumulants $\left\{r_{k}\right\}$ of $\left\{a_{i}\right\}_{i \in J}$, recursively by the following moment cumulant relation

$$
\tau\left(a_{i_{1}} a_{i_{2}} \ldots a_{i_{k}}\right)=\sum_{\pi \in E(k)} r_{\pi}\left(a_{i_{1}}, a_{i_{2}}, \ldots, a_{i_{k}}\right) .
$$

Observe that both sides of the moment cumulant relation above are equal to zero for odd values of $k$ and $\left\{r_{n}\right\}$ are multilinear maps.

For any self adjoint $a \in(\mathscr{A}, \tau)$, the half cumulants of $a$ are denoted by

$$
r_{n}(a)=r_{n}(a, a, \ldots, a), \quad n \geq 1 .
$$

Note that $r_{2 n+1}(a)=0$ for all $n \geq 0$. The half cumulant generating function of $a$ is defined as the formal power series

$$
H_{a}(t)=\sum_{n \geq 1}(-1)^{n} \frac{r_{2 n}(a)}{(n !)^{2}} t^{2 n} .
$$

The reason for the extra $n$ ! in the denominator shall be clear as we proceed.

Example 3. If the law of $a$ is the symmetrized Rayleigh law $R$, then it is easy to see that

$$
r_{2}(a)=1, \quad r_{2 n}(a)=0(\text { for } n>1) \text { and } H_{a}(t)=-t^{2} .
$$

Suppose $\left\{a_{i}\right\}$ are half independent. Using (6) recursively, it is easy to see that for any even $k>1$,

$$
r_{k}\left(a_{i_{1}}, a_{i_{2}}, \ldots, a_{i_{k}}\right)=0 \text { if } a_{i_{1}} a_{i_{2}} \ldots a_{i_{k}} \text { is unbalanced. }
$$

For example, let $\left\{a_{1}, a_{2}\right\} \in(\mathscr{A}, \tau)$, be half independent. Then

$$
r_{2}\left(a_{1}, a_{2}\right)=\tau\left(a_{1} a_{2}\right)=0 \text { and } r_{2}\left(a_{2}, a_{1}\right)=\tau\left(a_{2} a_{1}\right)=0 .
$$

Again using (6),

$$
\begin{aligned}
& r_{4}\left(a_{1}, a_{1}, a_{1}, a_{2}\right)=\tau\left(a_{1} a_{1} a_{1} a_{2}\right)-r_{2}\left(a_{1}, a_{1}\right) r_{2}\left(a_{1}, a_{2}\right)-r_{2}\left(a_{1}, a_{1}\right) r_{2}\left(a_{1}, a_{2}\right)=0, \\
& r_{4}\left(a_{1}, a_{2}, a_{1}, a_{2}\right)=\tau\left(a_{1} a_{2} a_{1} a_{2}\right)-r_{2}\left(a_{1}, a_{2}\right) r_{2}\left(a_{1}, a_{2}\right)-r_{2}\left(a_{1}, a_{2}\right) r_{2}\left(a_{1}, a_{2}\right)=0 .
\end{aligned}
$$

Classical independence and freeness are characterized by classical cumulants and free cumulants respectively (see Theorem 5.3.15 of Anderson et. al. (2009)[1] or Theorem 11.16 of Nica and Speicher (2006) [10] for the free cumulant results). The corresponding characterization for half independent random variables is the following: 
Theorem 1. Let $\left\{a_{i}\right\}_{1 \leq i \leq l}$ be a sequence of self adjoint half commuting random variables and suppose for all $1 \leq j \leq l, a_{j}$ occurs $k_{j}$ times in $a=a_{i_{1}} a_{i_{2}} \ldots a_{i_{2}}$.

(i) Suppose $\left\{a_{i}\right\}$ are half independent and $a$ is balanced with respect to $\left\{a_{i}\right\}_{1 \leq i \leq l}$. If $k_{i}, k_{j} \geq 2$ for some $1 \leq i, j \leq l$, then $r_{2 n}\left(a_{i_{1}}, a_{i_{2}}, \ldots, a_{i_{2 n}}\right)=0$.

(ii) If $\tau\left(a_{i_{1}} a_{i_{2}} \ldots a_{i_{k}}\right)=0$ whenever $k$ is odd and $r_{2 n}\left(a_{i_{1}}, a_{i_{2}}, \ldots, a_{i_{2 n}}\right)=0$ whenever $k_{i}, k_{j} \geq 1$ for some $1 \leq i, j \leq l$, then $\left\{a_{i}\right\}_{1 \leq i \leq l}$ are half independent.

Proof of the above theorem is a little long and we postpone it to Section 6.

An important consequence of Theorem 1 is the following corollary which is analogous to the additivity of cumulant generating function and $R$-transform for classical independence and free independence respectively.

Corollary 1. If $a$ and $b$ are self adjoint, half commuting and half independent then

$$
r_{2 n}(a+b)=r_{2 n}(a)+r_{2 n}(b) \text { for all } n \geq 1 \text {, and } H_{a+b}(t)=H_{a}(t)+H_{b}(t),
$$

where the second equality holds between formal power series.

Proof. Note that $r_{2}(a, b)=\tau(a b)=0$. Hence the result is true for $n=2$ since

$$
\begin{aligned}
r_{2}(a+b) & =r_{2}(a+b, a+b) \\
& =r_{2}(a, a+b)+r_{2}(b, a+b) \\
& =r_{2}(a, a)+r_{2}(a, b)+r_{2}(b, a)+r_{2}(b, b) \\
& =r_{2}(a)+r_{2}(b) .
\end{aligned}
$$

For $n>2$,

$$
\begin{aligned}
r_{2 n}(a+b) & =r_{2 n}(a+b, \ldots, a+b) \\
& =r_{2 n}(a, a+b, \ldots, a+b)+r_{2 n}(b, a+b, \ldots, a+b) \\
& =r_{2 n}(a, a \ldots, a)+r_{2 n}(b, b, \ldots, b)+\sum_{\left(a_{1}, a_{2}, \ldots a_{2 n}\right): a_{i} \in\{a, b\}, a_{i} \neq a \forall i} r_{2 n}\left(a_{1}, a_{2}, \ldots, a_{2 n}\right) .
\end{aligned}
$$

Now if $\left(a_{1}, a_{2}, \ldots, a_{2 n}\right)$ is unbalanced then by (10), $r_{2 n}\left(a_{1}, a_{2}, \ldots, a_{2 n}\right)=0$. If $\left(a_{1}, a_{2}, \ldots, a_{2 n}\right)$ is balanced but $a, b$ both appear in the tuple, then by Theorem 1 (i), $r_{2 n}\left(a_{1}, a_{2}, \ldots, a_{2 n}\right)=0$. So only the first two terms survive in the last expression and hence $r_{2 n}(a+b)=r_{2 n}(a)+r_{2 n}(b)$.

We now proceed to express the relation between moments and half cumulants of a random variable in terms of appropriate generating functions. This will leads us to the appropriate generating functions for arbitrary probability measures in the next section. Recall that from Definition 2,

$$
m_{2 n}(a)=\sum_{\pi \in E(2 n)} r_{\pi}(a)
$$

\section{Theorem 2.}

$$
m_{2 n}(a)=\sum_{s=0}^{n-1}\left(\begin{array}{l}
n \\
s
\end{array}\right)\left(\begin{array}{c}
n-1 \\
s
\end{array}\right) r_{2(n-s)}(a) m_{2 s}(a)
$$

Proof. We adapt the proof of Proposition 7.7 of Banica, Curran and Speicher (2009)[3] for the recursion formula for cardinality of $E(2 n)$. Fix any $\pi \in E(2 n)$. Then each partition block has same number of odd and even members. Now place the sequence of odd numbers in one row and place the even members in the next row. In this way, $E(2 n)$ is viewed as the set of partitions between the upper and lower rows of $n$ points such that each partition block has same number of upper and lower members. Now to form any such partition, we perform the following steps: 
- First pick a number $s \in\{1,2, \ldots, n\}$. This shall be the total number of odd elements in the partition block containing 1 .

- Connect 1 in the upper row to some other $(s-1)$ points in the upper row. So we have chosen all the odd members of the partition block that contains 1.

- Now choose $s$ points in the lower row and connect them to the already connected $s$ points in the upper row, thus completing the symmetric partition block of size $2 s$ containing 1 .

- Now partition the remaining $(n-s)$ odd points of the upper row and the remaining $(n-s)$ even points in the lower row in any symmetric way to essentially partition a set of size $2(n-s)$ in a symmetric way.

The number of such partitions is clearly $\left(\begin{array}{c}n-1 \\ s-1\end{array}\right)\left(\begin{array}{l}n \\ s\end{array}\right) \# E_{2(n-s)}$. Now observe that for any fixed $s$, the total contribution to $m_{2 n}(a)$ from all these partitions equals $r_{2 s}(a) m_{2(n-s)}(a)$. Hence

$$
\begin{aligned}
m_{2 n}(a) & =\sum_{s=1}^{n}\left(\begin{array}{c}
n-1 \\
s-1
\end{array}\right)\left(\begin{array}{l}
n \\
s
\end{array}\right) r_{2 s}(a) m_{2(n-s)}(a) \\
& =\sum_{s=0}^{n-1}\left(\begin{array}{c}
n-1 \\
s
\end{array}\right)\left(\begin{array}{l}
n \\
s
\end{array}\right) r_{2(n-s)}(a) m_{2 s}(a) .
\end{aligned}
$$

We now convert the above relation to a relation between appropriate generating functions of $\left\{m_{2 k}\right\}$ and $\left\{r_{2 k}\right\}$. We drop the argument $a$ in these expressions for ease of notation. Consider the following two formal power series:

$$
\tilde{T}(t)=\sum_{n=0}^{\infty} \frac{m_{2 n} t^{2 n}}{(n !)^{2}} \text { and } \tilde{H}(t)=\sum_{n=0}^{\infty} \frac{r_{2 n} t^{2 n}}{(n !)^{2}} .
$$

Their formal derivatives are:

$$
\tilde{T}^{\prime}(t)=2 \sum_{n=1}^{\infty} \frac{m_{2 n} t^{2 n-1}}{n !(n-1) !} \text { and } \tilde{H}^{\prime}(t)=2 \sum_{n=1}^{\infty} \frac{r_{2 n} t^{2 n-1}}{n !(n-1) !} .
$$

Corollary 2. The above formal power series are related by the following relation, $\tilde{T}^{\prime}(t)=\tilde{T}(t) \tilde{H}^{\prime}(t)$ where $\tilde{T}(0)=1$ and $\tilde{H}(0)=1$. When considered as appropriate functions, this differential equation has a solution $\tilde{T}(t)=\exp (\tilde{H}(t)-1)$. Hence, formally $\tilde{H}(t)=1+\log \tilde{T}(t)$.

Proof. Using Theorem 2,

$$
\begin{aligned}
\tilde{T}^{\prime}(t) & =2 \sum_{n=1}^{\infty} \frac{m_{2 n} t^{2 n-1}}{n !(n-1) !}=2 \sum_{n=1}^{\infty} \sum_{s=0}^{n-1} \frac{\left(\begin{array}{c}
n \\
s
\end{array}\right)\left(\begin{array}{c}
(n-1) \\
s
\end{array}\right)}{(n-s) !(n-s-1) !} r_{2(n-s)} m_{2 s} t^{2 n-1} . \\
& =2 \sum_{n=1}^{\infty} \sum_{s=0}^{n-1} \frac{1}{(s !)^{2} n !(n-1) !} r_{2(n-s)} m_{2 s} t^{2 n-1}=2 \sum_{s=0}^{\infty} \sum_{n=s+1}^{\infty} \frac{r_{2(n-s)} t^{2(n-s)-1}}{(n-s) !(n-s-1) !} \frac{m_{2 s} t^{2 s}}{(s !)^{2}} \\
& =2 \sum_{s=0}^{\infty} \sum_{l=1}^{\infty} \frac{r_{2 l} t^{2 l-1}}{l !(l-1) !} \frac{m_{2 s} t^{2 s}}{(s !)^{2}}=\tilde{T}(t) \tilde{H}^{\prime}(t) .
\end{aligned}
$$

Rest of the claims now follow easily. 


\section{T-transform}

In this section we introduce a new transform, which we call the $T$-transform, appropriate for the study of half independence. We show that the $T$-transform is very closely related to the characteristic function.

\subsection{T-transform for general probability measures}

The finiteness of the two power series in (11) impose growth conditions on the moments, thereby restricting the class of variables (or measures) for which the transforms are defined. We now remedy this.

When $a \in(\mathscr{A}, \tau)$ is a self adjoint random variable with a compactly supported law $\mu_{a}$, using Fubini's theorem,

$$
\tilde{T}(t)=\sum_{n=0}^{\infty} \frac{m_{2 n}(a) t^{2 n}}{(n !)^{2}}=\int_{\mathbb{R}} \sum_{n=0}^{\infty} \frac{(x t)^{2 n}}{(n !)^{2}} d \mu_{a}(x)=\int_{\mathbb{R}} I_{0}(2 x t) d \mu_{a}(x)
$$

where

$$
I_{0}(t)=\sum_{n=0}^{\infty} \frac{(t / 2)^{2 n}}{(n !)^{2}}=\frac{1}{\pi} \int_{0}^{\pi} \cosh (t \cos \theta) d \theta
$$

is the modified Bessel function of order 0 (see Section 3.7 of Watson (1995)[12]).

Suppose $\mu_{a}$ is symmetric about 0 . Let $\phi_{\mu_{a}}$ denote the characteristic function of $\mu_{a}$. Then we know that $\phi_{\mu_{a}}(t)$ is real. Using this and (12),

$$
\begin{aligned}
\tilde{T}(i t) & =\frac{1}{\pi} \int_{\mathbb{R}} \int_{0}^{\pi} \cosh (2 x i t \cos \theta) d \theta d \mu_{a}(x) \\
& =\frac{1}{\pi} \int_{0}^{\pi} \int_{\mathbb{R}} \cos (2 x t \cos \theta) d \mu_{a}(x) d \theta \\
& \left.=\frac{1}{\pi} \int_{0}^{\pi} \phi_{\mu_{a}}(2 t \cos \theta) d \theta \text { (since } \mu_{a} \text { is symmetric about } 0\right) .
\end{aligned}
$$

We have thus arrived at the following definition:

Definition 3. For any law $\mu$ which is symmetric about zero (but does not necessarily have compact support), define the transform,

$$
T_{\mu}(t)=\tilde{T}(i t)=\frac{1}{\pi} \int_{0}^{\pi} \phi_{\mu}(2 t \cos \theta) d \theta, t \in \mathbb{R} .
$$

We shall also use $T_{a}$ to denote the transform $T_{\mu_{a}}$. If $\mu$ is compactly supported or more generally if $m_{2 n}=\int_{\mathbb{R}} x^{2 n} d \mu(x) \leq C^{n} n$ ! for all $n$, then it easily follows from the above discussions that

$$
T_{\mu}(t)=\sum_{n=0}^{\infty} \frac{m_{2 n}(-1)^{n} t^{2 n}}{(n !)^{2}} \text { for all } t \in \mathbb{R}
$$

Example 4. If $\mu$ is symmetrized Rayleigh $R_{\sigma}$, then $m_{2 n}=\sigma^{2 n} n$ ! for all $n$ and hence

$$
T_{\mu}(t)=\sum_{n=0}^{\infty} \frac{m_{2 n}(-1)^{n} t^{2 n}}{(n !)^{2}}=\sum_{n=0}^{\infty} \frac{(-1)^{n}(\sigma t)^{2 n}}{n !}=\exp \left(-\sigma^{2} t^{2}\right), \quad t \in \mathbb{R} .
$$


We now connect $T$ and $\phi$ through product convolution. For probability measures $\mu$ on $\mathbb{R}$ and $\rho$ on $(0, \infty)$, we use the product convolution $\circledast$ where

$$
(\mu \circledast \rho)(B)=\int_{0}^{\infty} \mu\left(x^{-1} B\right) \rho(d x)=(\mu \otimes \rho) f^{-1}(B) \text { with } f(x, y)=x y,
$$

for any Borel set $B$. This gives a probability measure on $\mathbb{R}$ since $\mu\left(x^{-1} \mathbb{R}\right)=1$ for every $x \neq 0$. We skip the proof of the following Lemma.

Lemma 1. Let $\rho$ be the arcsine law with density $\frac{2}{\pi} \frac{1}{\sqrt{1-\alpha^{2}}}$ for $0<\alpha<1$. If $\mu$ is a probability measure on $\mathbb{R}$ which is symmetric around 0 , then

$$
T_{\mu}(t)=\phi_{\mu \circledast \rho}(2 t)
$$

Remark 2. Motivated by random walks with spherical symmetry, Kingman (1963) [8] (see Section 4) defined the radial characteristic function ${ }^{n} \psi_{X}(t)$ (in $n$ dimensions) of a nonnegative random variable $X$ with law $\mu$. Interestingly the final expression for $T$-transform is related to this:

$$
T_{\mu}(t)={ }^{n} \psi_{X}(2 t) \text { for } n=2
$$

Kingman showed that the radial characteristic function is measure determining and convergence determining for nonnegative random variables. Although we are dealing with measures symmetric about 0 , we can use Kingman's result to derive similar properties for T-transform.

The next Lemma follows easily from Lemma 2 and Theorem 2 of Kingman (1963) [8] respectively or it can be proved directly using the theory of Mellin transform and weak convergence.

Lemma 2. (i) Let $\mu$ and $v$ be two symmetric (about zero) probability measures. Then $T_{\mu}(t)=T_{v}(t)$ for all $t \in \mathbb{R}$ if and only if $\mu=v$.

(ii) Suppose $\left\{\mu_{n}\right\}$ and $\mu$ are symmetric probability measures. Then $\mu_{n}$ converges to $\mu$ weakly, if and only if $T_{\mu_{n}}(t)$ converges to $T_{\mu}(t)$ for all $t \in \mathbb{R}$.

Since $T_{\mu}(t)=\phi_{\mu \circledast \rho}(t)$ and $\phi$ is continuous at 0 , the logarithm of $T$ is well defined in a neighbourhood of 0 . This leads us to the following definition of half cumulant generating function which is more general than the one given in (8).

Definition 4. The half cumulant generating function of any symmetric probability measure $\mu$ is defined as

$$
H_{\mu}(t)=\log T_{\mu}(t) \text { (in an appropriate neighborhood of zero). }
$$

The half cumulant generating function $H_{a}$ of any $a$ in $(\mathscr{A}, \tau)$ is $H_{\mu_{a}}$ whenever $\mu_{a}$ exists and is symmetric.

Example 5. If $\mu$ is symmetrized Rayleigh $R_{\sigma}$, (see Example 4), then $T_{\mu}(t)=\exp \left(-\sigma^{2} t^{2}\right)$ is non-zero for all $t \in \mathbb{R}$. So

$$
H_{\mu}(t)=-\sigma^{2} t^{2} \text { for all } t \in \mathbb{R} .
$$

Conversely, if $H_{\mu}(t)=-\sigma^{2} t^{2}$, then $\mu$ is the symmetrized Rayleigh law $R_{\sigma}$. 


\subsection{Half convolution of two symmetric measures}

As mentioned before, there is no existing notion of half independence for unbounded operators. Here we do not give a definition for half independence for such operators but we define a suitable notion of half convolution for arbitrary probability measures with the help of the above transforms. We proceed as follows: suppose $(\mathscr{A}, \tau)$ is a $C^{*}$-probability space and $a, b \in(\mathscr{A}, \tau)$ are two self adjoint, half independent random variables with compactly supported measures $\mu_{a}$ and $\mu_{b}$ on $\mathbb{R}$ respectively. Then obviously, $\mu_{a}$ and $\mu_{b}$ are symmetric and in a neighbourhood of zero the following relations hold.

$$
H_{a+b}(t)=H_{a}(t)+H_{b}(t) \text { and } T_{a+b}(t)=T_{a}(t) T_{b}(t) .
$$

In this case the measure $\mu_{a+b}$ is the measure corresponding to the random variable $(a+b) \in(\mathscr{A}, \tau)$ and is compactly supported and symmetric. Note that from the second relation of (15) we have

$$
\phi_{\mu_{a+b} \circledast \rho}(t)=\phi_{\left(\mu_{a} \circledast \rho\right) *\left(\mu_{b} \circledast \rho\right)}(t)
$$

in a neighbourhood of zero. As the measures above are compactly supported the relation holds for all $t \in \mathbb{R}$ and hence $T_{a+b}(t)=T_{a}(t) T_{b}(t)$ for all $t \in \mathbb{R}$.

We now define the half convolution (notation: $\oslash$ ) of two arbitrary symmetric measures $\mu$ and $v$. First assume that $\mu$ and $v$ are compactly supported measures. Now using similar argument as given in Proposition 2.8 of Banica, Curran and Speicher (2010)[2], we can construct two half independent random variables $y_{1}, y_{2}$ as follows:

Let $X_{1}, X_{2}$ be two independent random variables such that $X_{1}$ and $X_{2}$ have law $\mu$ and $v$ respectively. Let $U_{1}, U_{2}$ be two independent Haar unitary random variables which are independent from $X_{1}, X_{2}$ and let $\xi_{i}=U_{i} X_{i}$. Then $\xi_{1}, \xi_{2}$ are independent and

$$
\begin{aligned}
\mathrm{E}\left[\xi_{i}^{n} \bar{\xi}_{i}^{m}\right]=\mathrm{E}\left[X_{i}^{n+m}\right] \mathrm{E}\left[U_{i}^{n} \bar{U}_{i}^{m}\right] & =\delta_{n m} \mathrm{E}\left[X_{i}^{n+m}\right] \\
& =\left\{\begin{array}{lll}
\mathrm{E}\left[X_{i}^{2 n}\right] & \text { if } & n=m \\
0 & \text { if } & n \neq m
\end{array}\right.
\end{aligned}
$$

Now define the variables $y_{1}, y_{2}$ as

$$
y_{i}=\left(\begin{array}{ll}
0 & \xi_{i} \\
\bar{\xi}_{i} & 0
\end{array}\right) .
$$

Then it is easy to check using (16) that $y_{1}, y_{2}$ are half independent and have laws $\mu, v$ respectively. Then from Lemma 1

$$
T_{y_{1}}(t)=T_{\mu}(t)=\phi_{\mu \circledast \rho}(2 t) \text { and } T_{y_{2}}(t)=T_{v}(t)=\phi_{v \circledast \rho}(2 t) .
$$

Consider the product $T_{\mu}(t) T_{v}(t)$. Then due to relation (15),

$$
T_{\mu}(t) T_{v}(t)=T_{y_{1}}(t) T_{y_{2}}(t)=T_{y_{1}+y_{2}}(t)=T_{\beta}(t)=\phi_{\beta \circledast \rho}(2 t),
$$

where $\beta$ is a symmetric measure corresponding to random variable $\left(y_{1}+y_{2}\right)$ and this is unique by Lemma 2 (i). We define $\beta$ as half convolution of $\mu$ and $v$, that is,

$$
\mu \oslash v:=\beta \text {. }
$$

Now suppose $\mu$ and $v$ are two arbitrary symmetric measure. Define for $n \in \mathbb{N}$, the two measures $\mu_{n}$ and $v_{n}$ by

$$
\mu_{n}(B)=\mu(B \cap[-n, n]) \text { and } v_{n}(B)=v(B \cap[-n, n]) \text {, for any Borel set } B \subseteq \mathbb{R} .
$$


Then $\mu_{n}$ and $v_{n}$ are compactly supported symmetric measure on $\mathbb{R}$ and $\mu_{n}, v_{n}$ converges weakly to $\mu, v$ respectively. Then as before we have measures $\beta_{n}$ such that

$$
T_{\mu_{n}}(t) T_{v_{n}}(t)=\phi_{\mu_{n} \circledast \rho}(2 t) \phi_{v_{n} \circledast \rho}(2 t)=\phi_{\beta_{n} \circledast \rho}(2 t) .
$$

Since $\mu_{n}, v_{n}$ converges weakly to $\mu, v$ respectively, $\phi_{\mu_{n} \circledast \rho}(2 t) \phi_{v_{n} \circledast \rho}(2 t)$ converges and hence $\phi_{\beta_{n} \circledast \rho}(2 t)$ converges for all $t$. Hence $\beta_{n} \circledast \rho$ converges weakly to some measure on $\mathbb{R}$. It easily follows that $\left\{\beta_{n}\right\}$ is tight and $\beta_{n} \circledast \rho$ converges weakly to $\beta \circledast \rho$ for some unique measure $\beta$. Uniqueness of $\beta$ follows from Lemma 2(i) and we have $T_{\mu}(t) T_{v}(t)=T_{\beta}(t)$ for all $t \in \mathbb{R}$. Hence we arrive at the following definition.

Definition 5. For any two symmetric probability measures $\mu$ and $v$, their half independent convolution is defined as the unique measure $\beta$ such that $T_{\mu}(t) T_{v}(t)=T_{\beta}(t)$ for all $t \in \mathbb{R}$. We write

$$
\mu \oslash v:=\beta \text {. }
$$

Note that $\oslash$ is associative and commutative on the space of symmetric measures.

Remark 3. This convolution is quite similar to the notion of radial sum in $n$ dimensions of two independent nonnegative random variables $X, Y$ defined in Section 3 of Kingman (1963) [8].

\section{Some properties of half independent convolution}

In this section, we establish the CLT and Cramer's theorem for half independent convolutions. For clarity, we present both, the algebraic version and the measure version in each case. First the Cramer's theorem.

Theorem 3. (i) Let $a$ and $b$ be self adjoint and half independent random variables of $a$ *-probability space $(\mathscr{A}, \tau)$ where $\tau$ is a state. Suppose $\mu_{\frac{a+b}{\sqrt{2}}}$ is $R_{\sigma}$. Then $\mu_{a}$ and $\mu_{b}$ exist, and are symmetrized Rayleigh.

(ii) If $\mu$ and $v$ are symmetric probability measures such that $\mu \oslash v$ is symmetrized Rayleigh, then both $\mu$ and $v$ are symmetrized Rayleigh.

Proof. (i) Without loss, assume that $\sigma=1$. Then

$$
\tau\left(((a+b) / \sqrt{2})^{2 k}\right)=k !
$$

From this, and half independence, it is easy to see that

$$
\tau\left(a^{2 k+1}\right)=0 \text { and } \tau\left(a^{2 k}\right) \leq 2^{k} k !
$$

Thus there exists unique symmetric probability measures $\mu_{a}$ and $\mu_{b}$ such that

$$
\tau\left(a^{k}\right)=\int x^{k} d \mu_{a}(x) \text { and } \tau\left(b^{k}\right)=\int x^{k} d \mu_{b}(x) .
$$

It easily follows that the characteristic functions of $\mu_{a}$ and $\mu_{b}$ are real and hence they are symmetric about origin. Hence for all $t$,

$$
\left(T_{\frac{a}{\sqrt{2}}}(t) T_{\frac{b}{\sqrt{2}}}(t)\right)=\exp \left(-t^{2}\right) .
$$


So we have

$$
\left(\phi_{\mu_{a} \circledast \rho}(2 t)\right)\left(\phi_{\mu_{b} \circledast \rho}(2 t)\right)=\exp \left(-t^{2}\right) .
$$

Hence, by the classical Cramer's theorem it follows that each of the product on the left side must be the characteristic function of a normal distribution. So $T_{\frac{a}{\sqrt{2}}}(t)=\exp \left(-\frac{t^{2}}{\sigma^{2}}\right)$ for some $\sigma$. As a consequence, by the uniqueness Lemma 2, $a$ is symmetrized Rayleigh $R_{\sqrt{2} / \sigma}$. Likewise, $b$ is symmetrized Rayleigh $R_{\sqrt{2} / \sigma^{\prime}}$.

The proof of the second part follows easily from the above arguments.

The next result is a CLT for half independent random variables in a noncommutative *-probability space $(\mathscr{A}, \tau)$. It is relevant to recall that the notion of half independence holds in any such space. However, the corresponding probability laws may not exist and hence the developments of the previous section do not apply. As a consequence, the proof of this theorem is based solely on Definition 1 and counting via symmetric partitions.

Theorem 4. Let $\left\{x_{i}\right\}$ be a sequence of self adjoint half independent random variables in $(\mathscr{A}, \tau)$ with $\tau\left(x_{i}\right)=0$ and $\tau\left(x_{i}^{2}\right)=1$ and $\sup _{i} \tau\left(x_{i}^{k}\right)<\infty$ for every $k$. If $S_{n}=x_{1}+x_{2}+\cdots+x_{n}$, then $S_{n} / \sqrt{n}$ converges in law to the standard symmetrized Rayleigh distribution $R$.

Proof. To show the required convergence it is enough to show that,

$$
\frac{\tau\left(S_{n}^{2 k}\right)}{n^{k}} \rightarrow k ! \text { and } \frac{\tau\left(S_{n}^{2 k+1}\right)}{n^{k+1 / 2}} \rightarrow 0 .
$$

By applying half independence and unbalancedness of each term in the expansion of $\left(x_{1}+\cdots+\right.$ $\left.x_{n}\right)^{2 k+1}$, it immediately follows that $\tau\left(\left(x_{1}+\cdots+x_{n}\right)^{2 k+1}\right)=0$. It is thus enough to consider the even moments.

Consider any $q=x_{i_{1}} \cdots x_{i_{2 k}}$ in the expansion of $\left(x_{1}+\cdots+x_{n}\right)^{2 k}$ which is unbalanced. Then by half independence $\tau(q)=0$. Thus we are left with only balanced monomials $q$. We divide such monomials into two sets:

$M_{1}=\left\{x_{i_{1}} \cdots x_{i_{2 k}} ; i_{j} \in\{1,2, \cdots, n\}\right.$ balanced and every random variable appears exactly twice $\}$, $M_{2}=\left\{x_{i_{1}} \cdots x_{i_{2 k}} ; i_{j} \in\{1,2, \cdots, n\}\right.$ balanced and at least one random variable appears more than twice $\}$.

Observe that $M_{2}$ has at most $(k-1)$ many distinct random variables and hence

$$
\# M_{2} \leq C_{k} n(n-1) \cdots(n-k+2),
$$

for some constant $C_{k}$ that depends only on $k$. Now since all moments are finite,

$$
\frac{1}{n^{k}} \sum_{M_{2}} \tau\left(x_{i_{1}} \cdots x_{i_{2 k}}\right) \leq C_{k}^{\prime} \frac{n(n-1) \cdots(n-k+2)}{n^{k}} \rightarrow 0 \text { as } n \rightarrow \infty .
$$

Now consider $M_{1}$. Pick a fixed set of $k$ variables from $\left\{x_{1}, \ldots, x_{n}\right\}$. Then there are exactly $k$ ! ways of forming a monomial of length $2 k$ of these $k$ variables where each of the variables appears exactly once in an odd position and once in an even position. Hence

$$
\# M_{1}=k ! \times n(n-1) \cdots(n-k+1) .
$$

Therefore as $n \rightarrow \infty$

$$
\frac{1}{n^{k}} \sum_{M_{1}} \tau\left(x_{i_{1}} \cdots x_{i_{2 k}}\right)=\frac{\# M_{1}}{n^{k}} \tau\left(x_{1}^{2} \cdots x_{k}^{2}\right)=\frac{k ! \times n(n-1) \cdots(n-k+1)}{n^{k}} \rightarrow k ! .
$$

Hence the result follows. 
The CLT for half independent convolution of measures is stated next. Its proof is easy and is in spirit similar to the proof of Theorem 6 in Kingman (1963)[8].

Theorem 5. Let $\left\{\mu_{n}\right\}$ be a sequence of symmetric probability measures with variance 1 . Suppose for every $\epsilon>0$,

$$
\frac{1}{n} \sum_{k=1}^{n} \int_{\{x:|x| \geq \epsilon \sqrt{n}\}} x^{2} d \mu_{k}(x) \rightarrow 0 \text { as } n \rightarrow \infty \text { (Lindeberg's condition). }
$$

Then

$$
\delta_{n}:=D_{\frac{1}{\sqrt{n}} \mu_{1}} \oslash D_{\frac{1}{\sqrt{n}} \mu_{2}} \oslash \cdots \oslash D_{\frac{1}{\sqrt{n}} \mu_{n}}
$$

converges weakly to $R$ where $D_{c \mu_{i}}(B)=\mu_{i}\left(c^{-1} B\right)$ for any Borel set $B$ in $\mathbb{R}$.

Proof. By definition of half convolution

$$
\begin{aligned}
T_{\delta_{n}}(t) & =T_{D_{\frac{1}{\sqrt{n}} \mu_{1}}}(t) T_{D_{\frac{1}{\sqrt{n}} \mu_{2}}}(t) \cdots T_{D_{\frac{1}{\sqrt{n}} \mu_{n}}}(t) \\
& =\phi_{D_{\frac{1}{\sqrt{n}} \mu_{1}} \circledast \rho}(2 t) \phi_{D_{\frac{1}{\sqrt{n}} \mu_{2}} \circledast \rho}(2 t) \cdots \phi_{D_{\frac{1}{\sqrt{n}} \mu_{n}} \circledast \rho}(2 t),
\end{aligned}
$$

where $\rho$ is as defined in Lemma 1. Note that for any $\epsilon>0$

$$
\begin{aligned}
\frac{1}{n} \sum_{k=1}^{n} \int_{\{x:|x| \geq \epsilon \sqrt{n}\}} x^{2} d\left(\mu_{k} \circledast \rho\right)(x) & =\frac{1}{n} \sum_{k=1}^{n} \frac{2}{\pi} \int_{0}^{1} \int_{\{x:|x \alpha| \geq \epsilon \sqrt{n\}}} \frac{x^{2} \alpha^{2}}{\sqrt{1-\alpha^{2}}} \mu_{k}(x) d \alpha \\
& =\frac{2}{\pi} \int_{0}^{1} \frac{\alpha^{2}}{\sqrt{1-\alpha^{2}}}\left[\frac{1}{n} \sum_{k=1}^{n} \int_{\{x:|x \alpha| \geq \epsilon \sqrt{n}\}} x^{2} d \mu_{k}(x)\right] d \alpha(19)
\end{aligned}
$$

Now as $n \rightarrow \infty$, last expression in (19) goes to 0 by dominated convergence theorem and condition (18). Hence the sequence of measure $\left\{\mu_{n} \circledast \rho\right\}$ satisfies Lindeberg's condition. Also

$$
\int_{0}^{1} x^{2} d\left(\mu_{k} \circledast \rho\right)(x)=\frac{2}{\pi} \int_{0}^{1} \frac{1}{\sqrt{1-\alpha^{2}}} \int_{\mathbb{R}} x^{2} \alpha^{2} d \mu_{k}(x) d \alpha=\frac{1}{2} .
$$

Hence by classical central limit theorem (see Billingsley (1995)[6]) as $n \rightarrow \infty$,

$$
T_{\delta_{n}}(t) \rightarrow \phi_{N_{0, \frac{1}{2}}}(t)=\exp \left(-t^{2}\right)
$$

where $N_{0, \frac{1}{2}}$ is the Gaussian measure with mean zero and variance $1 / 2$. We know $T_{R}(t)=\exp \left(-t^{2}\right)$. Hence $\delta_{n}$ converges weakly to the standard (symmetrized) Rayleigh measure $R$.

\section{Proof of Theorem 1}

Proof. (i) For convenience, denote the trivial partition of $\{1,2, \ldots, 2 n\}$ with one single block by $I_{2 n}$. We prove the first part of the theorem through induction on $d$ (which equals the number of distinct random variables in $a=a_{i_{1}} a_{i_{2}} \ldots a_{i_{2 n}}$ ) and $n$. For each fixed values of $d$ we use induction on $n$. We use notation $D_{1}$ for induction on $d$ and $D_{2}$ for induction on $n$. 
Now from relation (6),

$$
\tau\left(a_{i_{1}} a_{i_{2}} \ldots a_{i_{2 n}}\right)=\sum_{\pi \in E(2 n)} r_{\pi}\left(a_{i_{1}}, a_{i_{2}}, \ldots, a_{i_{2 n}}\right) .
$$

If $\pi \in E(2 n)$ has a block $V$ such that $\left(a_{j_{1}} a_{j_{2}} \ldots a_{j_{|V|}}\right)$ is unbalanced, then by observation (10)

$$
r_{\pi}\left(a_{i_{1}}, a_{i_{2}}, \ldots, a_{i_{2 n}}\right)=0
$$

Therefore relation (20) reduces to

$$
\tau\left(a_{i_{1}} a_{i_{2}} \ldots a_{i_{2 n}}\right)=\sum_{\pi \in E^{\prime}(2 n)} r_{\pi}\left(a_{i_{1}}, a_{i_{2}}, \ldots, a_{i_{2 n}}\right)
$$

where

$$
E^{\prime}(2 n)=\left\{\pi \in E_{2 n}: \text { for each block } V \text { of } \pi, a_{j_{1}} a_{j_{2}} \ldots a_{j_{|V|}} \text { is balanced }\right\} \text {. }
$$

Note that $E^{\prime}(2 n)$ depends on the considered tuple $\left(a_{i_{1}}, a_{i_{2}}, \ldots, a_{i_{2 n}}\right)$. We shall use relation (21) repeatedly in the proof.

First assume $d=2$. Consider $\left(a_{i_{1}}, a_{i_{2}}, \ldots, a_{i_{2 n}}\right)$ where $a_{i_{j}} \in\left\{a_{1}, a_{2}\right\}$ and $k_{1}, k_{2} \geq 2$. So the minimum possible value of $n$ is 2 . For $n=2$, using (21), half commutativity and half independence,

$$
\begin{aligned}
r_{4}\left(a_{i_{1}}, a_{i_{2}}, a_{i_{3}}, a_{i_{4}}\right) & =\tau\left(a_{i_{1}} a_{i_{2}} a_{i_{3}} a_{i_{4}}\right)-\sum_{\pi \in E^{\prime}(4)-I_{4}} r_{\pi}\left(a_{i_{1}}, a_{i_{2}}, a_{i_{3}}, a_{i_{4}}\right) \\
& =\tau\left(a_{1}^{2}\right) \tau\left(a_{2}^{2}\right)-r_{2}\left(a_{1}, a_{1}\right) r_{2}\left(a_{2}, a_{2}\right) \\
& =\tau\left(a_{1}^{2}\right) \tau\left(a_{2}^{2}\right)-\tau\left(a_{1}^{2}\right) \tau\left(a_{2}^{2}\right)=0 .
\end{aligned}
$$

Now assuming that the result is true up to $(n-1)$, we prove it for any $n$ (induction $D_{2}$ ). Again by (21) and half independence,

$$
\begin{aligned}
r_{2 n}\left(a_{i_{1}}, a_{i_{2}}, \ldots, a_{i_{2 n}}\right) & =\tau\left(a_{i_{1}} a_{i_{2}} \ldots a_{i_{2 n}}\right)-\sum_{\pi \in E^{\prime}(2 n)-I_{2 n}} r_{\pi}\left(a_{i_{1}}, a_{i_{2}}, \ldots, a_{i_{2 n}}\right) \\
& =\tau\left(a_{1}^{2 k_{1}}\right) \tau\left(a_{2}^{2 k_{2}}\right)-\sum_{\pi \in E^{\prime}(2 n)-I_{2 n}} r_{\pi}\left(a_{i_{1}}, a_{i_{2}}, \ldots, a_{i_{2 n}}\right) .
\end{aligned}
$$

Let

$$
J_{j}=\left\{j_{1}, j_{2}, \ldots, j_{2 k_{j}}\right\}
$$

be the positions of $a_{j}$ for $j=1,2$ in $\left(a_{i_{1}}, a_{i_{2}}, \ldots, a_{i_{2 n}}\right)$. Define

$$
\Pi=\left\{\pi \in E^{\prime}(2 n)-I_{2 n} \text { : if } V \text { is a block in } \pi \text { then either } V \subset J_{1} \text { or } V \subset J_{2}\right\} \text {. }
$$

Note that if $\pi \in E^{\prime}(2 n)-\Pi$, then using (2) and induction $D_{2}, r_{\pi}\left(a_{i_{1}}, \ldots, a_{i_{2 n}}\right)=0$. Hence

$$
r_{2 n}\left(a_{i_{1}}, a_{i_{2}}, \ldots, a_{i_{2 n}}\right)=\tau\left(a_{1}^{2 k_{1}}\right) \tau\left(a_{2}^{2 k_{2}}\right)-\sum_{\pi \in \Pi} r_{\pi}\left(a_{i_{1}}, a_{i_{2}}, \ldots, a_{i_{2 n}}\right) .
$$

Since $a_{i_{1}} a_{i_{2}} \ldots a_{i_{2 n}}$ is balanced, $J_{j}, j=1,2$ are symmetric. Hence if $\Pi_{j}$ denotes all symmetric partitions of $\left\{j_{1}, \ldots, j_{2 k_{j}}\right\}$ then it is in bijection with $E\left(2 k_{j}\right)$. That is

$$
\Pi_{j}:=E\left\{j_{1}, \ldots, j_{2 k_{j}}\right\} \simeq E\left(2 k_{j}\right) \text { for } j=1,2
$$


Now observe that for any $\pi \in \Pi$ there exist unique $\sigma=\left\{V_{1}, V_{2}, \ldots V_{l}\right\} \in \Pi_{1}$ and unique $\eta=$ $\left\{U_{1}, U_{2}, \ldots U_{s}\right\} \in \Pi_{2}$ where $V_{i}, U_{i}$ are blocks such that $\pi=\left\{V_{1}, V_{2}, \ldots V_{l}, U_{1}, U_{2}, \ldots U_{s}\right\}:=\sigma \sqcup \eta$. Thus there is a bijection between $\Pi$ and $\Pi_{1} \star \Pi_{2}:=\left\{\sigma \sqcup \eta: \sigma \in \Pi_{1}\right.$ and $\left.\eta \in \Pi_{2}\right\}$. Therefore

$$
\begin{aligned}
r_{2 n}\left(a_{i_{1}}, a_{i_{2}}, \ldots, a_{i_{2 n}}\right) & =\tau\left(a_{1}^{2 k_{1}}\right) \tau\left(a_{2}^{2 k_{2}}\right)-\sum_{\pi \in \Pi} r_{\pi}\left(a_{i_{1}}, a_{i_{2}}, \ldots, a_{i_{2 n}}\right) \\
& =\tau\left(a_{1}^{2 k_{1}}\right) \tau\left(a_{2}^{2 k_{2}}\right)-\sum_{\sigma \in \Pi_{1}, \eta \in \Pi_{2}} r_{\sigma \sqcup \eta}\left(a_{i_{1}}, a_{i_{2}}, \ldots, a_{i_{2 n}}\right) \\
& =\tau\left(a_{1}^{2 k_{1}}\right) \tau\left(a_{2}^{2 k_{2}}\right)-\sum_{\sigma \in \Pi_{1}, \eta \in \Pi_{2}} r_{\sigma}\left(a_{1_{1}}, a_{1_{2}}, \ldots, a_{1_{2 k_{1}}}\right) r_{\eta}\left(a_{2_{1}}, a_{2_{2}}, \ldots, a_{2_{2 k_{2}}}\right) \\
& =\tau\left(a_{1}^{2 k_{1}}\right) \tau\left(a_{2}^{2 k_{2}}\right)-\sum_{\sigma \in E\left(2 k_{1}\right), \eta \in E\left(2 k_{2}\right)} r_{\sigma}\left(a_{1}, a_{1}, \ldots, a_{1}\right) r_{\eta}\left(a_{2}, a_{2}, \ldots, a_{2}\right) \\
& =\tau\left(a_{1}^{2 k_{1}}\right) \tau\left(a_{2}^{2 k_{2}}\right)-\left[\sum_{\sigma \in E\left(2 k_{1}\right)} r_{\sigma}\left(a_{1}, a_{1}, \ldots, a_{1}\right)\right]\left[\sum_{\eta \in E\left(2 k_{2}\right)} r_{\eta}\left(a_{2}, a_{2}, \ldots, a_{2}\right)\right] \\
& \left.=\tau\left(a_{1}^{2 k_{1}}\right) \tau\left(a_{2}^{2 k_{2}}\right)-\tau\left(a_{1}^{2 k_{1}}\right) \tau\left(a_{2}^{2 k_{2}}\right)=0 \quad \text { using }(6)\right) .
\end{aligned}
$$

Hence the result is true for $d=2$.

Now assuming that the result is true for $m$ many random variables where $m \leq d-1$, we shall prove the result for $m=d$ (induction $D_{1}$ ). For $m=d$, the minimum possible value of $n$ is $d$ and each $k_{j}=2$ for $1 \leq j \leq d$. So for $n=d$,

$$
\begin{aligned}
r_{2 d}\left(a_{i_{1}}, a_{i_{2}}, \ldots, a_{i_{2 d}}\right) & =\tau\left(a_{i_{1}} a_{i_{2}} \ldots a_{i_{2 d}}\right)-\sum_{\pi \in E^{\prime}(2 d)-I_{2 d}} r_{\pi}\left(a_{i_{1}}, a_{i_{2}}, \ldots, a_{i_{2 d}}\right) \\
& =\tau\left(a_{1}^{2}\right) \tau\left(a_{2}^{2}\right) \cdots \tau\left(a_{d}^{2}\right)-\sum_{\pi \in E^{\prime}(2 d)-I_{2 d}} r_{\pi}\left(a_{i_{1}}, a_{i_{2}}, \ldots, a_{i_{2 d}}\right) .
\end{aligned}
$$

Now observe that if $\pi \in E^{\prime}(2 d)-I_{2 d}$ then either $\pi$ has a block $V$ such that $4 \leq \# V \leq 2(d-1)$ or each block of $\pi$ has size 2 . For the first case, by definition of cumulant (2) and induction $D_{1}$,

$$
r_{\pi}\left(a_{i_{1}}, a_{i_{2}}, \ldots, a_{i_{2 d}}\right)=0 .
$$

So in (24), we are left with partitions $\pi \in E^{\prime}(2 d)-I_{2 d}$ whose each partition block is of size 2 . Hence

$$
\begin{aligned}
r_{2 d}\left(a_{i_{1}}, a_{i_{2}}, \ldots, a_{i_{2 d}}\right) & =\tau\left(a_{1}^{2}\right) \cdots \tau\left(a_{d}^{2}\right)-\sum_{\substack{\pi \in E^{\prime}(2 d) \\
\pi=\left\{V_{1}, \ldots, V_{d}\right\}, \# V_{i}=2 \forall i}} r_{\pi}\left(a_{i_{1}}, \ldots, a_{i_{2 d}}\right) \\
& =\tau\left(a_{1}^{2}\right) \cdots \tau\left(a_{d}^{2}\right)-r_{2}\left(a_{1}, a_{1}\right) \cdots r_{2}\left(a_{d}, a_{d}\right) \\
& =\tau\left(a_{1}^{2}\right) \cdots \tau\left(a_{d}^{2}\right)-\tau\left(a_{1}^{2}\right) \cdots \tau\left(a_{d}^{2}\right)=0 .
\end{aligned}
$$

So we have established the result for $n=d$.

Still holding $m=d$ and assuming that the result is true up to $(n-1)$, we now prove it for $n$. Let $a_{i_{1}} a_{i_{2}} \ldots a_{i_{2 n}}$ be balanced. Since we have $d$ distinct random variables, each $k_{j} \geq 2$ for $1 \leq j \leq d$. As before, let $J_{j}=\left\{j_{1}, j_{2}, \ldots, j_{2 k_{j}}\right\}$ be the positions of $a_{j}$ in $\left(a_{i_{1}}, a_{i_{2}}, \ldots, a_{i_{2 n}}\right)$ for $1 \leq j \leq d$. By similar argument as given in $d=2$ case, each $J_{j}$ is symmetric. Now

$$
r_{2 n}\left(a_{i_{1}}, a_{i_{2}}, \ldots, a_{i_{2 n}}\right)=\tau\left(a_{i_{1}} a_{i_{2}} \ldots a_{i_{2 n}}\right)-\sum_{\pi \in E^{\prime}(2 n)-I_{2 n}} r_{\pi}\left(a_{i_{1}}, a_{i_{2}}, \ldots, a_{i_{2 n}}\right)
$$




$$
=\tau\left(a_{1}^{2 k_{1}}\right) \tau\left(a_{2}^{2 k_{2}}\right) \cdots \tau\left(a_{d}^{2 k_{d}}\right)-\sum_{\pi \in E^{\prime}(2 n)-I_{2 n}} r_{\pi}\left(a_{i_{1}}, a_{i_{2}}, \ldots, a_{i_{2 n}}\right) .
$$

Suppose $\pi \in E^{\prime}(2 n)-I_{2 n}$ has $t(\geq 2)$ many blocks, $V_{1}, \ldots, V_{t}$. Suppose for some $s, 1 \leq s \leq t$; $V_{s} \cap J_{m} \neq \emptyset$ for at least two values of $m$, where $1 \leq m \leq d$. If number of such $m$ is strictly less than $d$, then by induction $D_{1}$ and Definition (2), $r_{\pi}\left(a_{i_{1}}, a_{i_{2}}, \ldots, a_{i_{2 n}}\right)=0$. If number of such $m$ is equal to $d$, then by induction $D_{2}$ (since, $\# V<2(n-1)$ ) and $(2), r_{\pi}\left(a_{i_{1}}, a_{i_{2}}, \ldots, a_{i_{2 n}}\right)=0$. So we are left with only $\pi \in \Pi$ where $\Pi$ is as defined below. Let

$$
\begin{aligned}
\Pi & =\left\{\pi \in E^{\prime}(2 n)-I_{2 n}: \pi=\left\{V_{1}, \ldots, V_{l}\right\} \text { and for all } i, V_{i} \subset J_{r} \text { for some } 1 \leq r \leq d\right\} \\
\Pi_{j} & =E\left(j_{1}, j_{2}, \ldots, j_{2 k_{j}}\right) \simeq E\left(2 k_{j}\right), 1 \leq j \leq d .
\end{aligned}
$$

Then following the argument given for $d=2$ case, we have

$$
\begin{aligned}
r_{2 n}\left(a_{i_{1}}, a_{i_{2}}, \ldots, a_{i_{2 n}}\right) & =\tau\left(a_{1}^{2 k_{1}}\right) \cdots \tau\left(a_{d}^{2 k_{d}}\right)-\sum_{\pi \in \Pi} r_{\pi}\left(a_{i_{1}}, a_{i_{2}}, \ldots, a_{i_{2 n}}\right) \\
& =\tau\left(a_{1}^{2 k_{1}}\right) \cdots \tau\left(a_{d}^{2 k_{d}}\right)-\sum_{\pi_{1} \in \Pi_{1}, \ldots, \pi_{d} \in \Pi_{d}} r_{\pi_{1} \sqcup \cdots \sqcup \pi_{d}}\left(a_{i_{1}}, a_{i_{2}}, \ldots, a_{i_{2 n}}\right) \\
& =\tau\left(a_{1}^{2 k_{1}}\right) \cdots \tau\left(a_{d}^{2 k_{d}}\right)-\sum_{\pi_{1} \in \Pi_{1}, \ldots, \pi_{d} \in \Pi_{d}} \prod_{j=1}^{d} r_{\pi_{j}}\left(a_{j_{1}}, a_{j_{2}}, \ldots, a_{j_{2 k_{j}}}\right) \\
& =\tau\left(a_{1}^{2 k_{1}}\right) \cdots \tau\left(a_{d}^{2 k_{d}}\right)-\prod_{j=1}^{d}\left[\sum_{\pi_{j} \in \Pi_{j}} r_{\pi_{j}}\left(a_{j_{1}}, a_{j_{2}}, \ldots, a_{j_{2 k_{j}}}\right)\right] \\
& =\tau\left(a_{1}^{2 k_{1}}\right) \cdots \tau\left(a_{d}^{2 k_{d}}\right)-\prod_{j=1}^{d}\left[\sum_{\pi \in E\left(2 k_{j}\right)} r_{\pi}\left(a_{j}, a_{j}, \ldots, a_{j}\right)\right] \\
& =\tau\left(a_{1}^{2 k_{1}}\right) \cdots \tau\left(a_{d}^{2 k_{d}}\right)-\tau\left(a_{1}^{2 k_{1}}\right) \cdots \tau\left(a_{d}^{2 k_{d}}\right)=0 .
\end{aligned}
$$

Hence the first part of the Theorem is proved.

(ii) For the second part observe that the definition of half cumulant reduces to the definition of half-liberated cumulant given in Banica, Curran and Speicher (2010)[2]. They showed in Theorem 2.11 that half-liberated cumulant characterizes half independence for half commuting random variables $\left\{x_{i}\right\} \in(\mathscr{A}, \tau)$. In particular if $\tau\left(x_{i_{1}} \cdots x_{i_{k}}\right)=0$ when $k$ is odd and $r_{\pi}\left(x_{i_{1}}, \cdots, x_{i_{k}}\right)=0$ whenever $\pi \in E(2 k)$ is not symmetric with respect to $i=\left(i_{1}, i_{2}, \cdots, i_{k}\right)$ (that is, it is not the case that $s$ and $t$ are in the same block of $\pi$ and $i_{s}=i_{t}$ ) then $\left\{x_{i}\right\}$ are half independent. Now these two conditions follow from our assumptions.

Remark 4. An anonymous Referee has pointed out that the proof of Theorem 1(i) maybe significantly shortened by using the the machinery of Lehner (2004) [9]. In fact, it turns out that by using the properties of Mobius function,

$$
r_{k}\left(a_{i_{1}}, a_{i_{2}}, \ldots, a_{i_{k}}\right)=\sum_{\pi \in E(k)}(-1)^{|\pi|-1}(|\pi|-1) ! \tau_{\pi}\left(a_{i_{1}}, a_{i_{2}}, \cdots, a_{i_{k}}\right) .
$$

From this, the characterisation of the half independence via the half cumulants can also be derived.

Acknowledgement. We are grateful to the two anonymous Referees for their constructive and insightful comments. We are also indebted to Steven Miller for his detailed comments. 


\section{References}

[1] G.W. Anderson, A. Guionnet, and O. Zeitouni. An introduction to random matrices. Cambridge University Press, 2010. MR2760897

[2] T. Banica, S. Curran, and R. Speicher. De Finetti theorems for easy quantum groups. Arxiv preprint arXiv:0907.3314, 2009.

[3] Teodor Banica, Stephen Curran, and Roland Speicher. Classification results for easy quantum groups. Pacific J. Math., 247(1):1-26, 2010. MR2718205

[4] H. Bercovici and D. Voiculescu. Free convolution of measures with unbounded support. Indiana Univ. Math. J., 42(3):733-773, 1993. ISSN 0022-2518. http://dx.doi.org/10. 1512/iumj.1993.42.42033. MR1254116

[5] H. Bercovici and D. Voiculescu. Superconvergence to the central limit and failure of the Cramér theorem for free random variables. Probab. Theory Related Fields, 103(2):215-222, 1995. MR1355057

[6] Patrick Billingsley. Probability and measure. Wiley Series in Probability and Mathematical Statistics. John Wiley \& Sons Inc., New York, third edition, 1995. A Wiley-Interscience Publication. MR1324786

[7] Arup Bose, Rajat Subhra Hazra, and Koushik Saha. Convergence of joint moments for independent random patterned matrices. To appear in Ann. Probab., 2010. MR2670197

[8] J. F. C. Kingman. Random walks with spherical symmetry. Acta Math., 109:11-53, 1963. MR0149567

[9] Franz Lehner. Cumulants in noncommutative probability theory. I.Noncommutative exchangeability systems. Math. Z., 248:67-100, 2004. http://dx.doi.org/10.1007/ s00209-004-0653-0 MR2092722

[10] Alexandru Nica and Roland Speicher. Lectures on the combinatorics of free probability, volume 335 of London Mathematical Society Lecture Note Series. Cambridge University Press, Cambridge, 2006. MR2266879

[11] Roland Speicher. On universal products. In Free probability theory (Waterloo, ON, 1995), volume 12 of Fields Inst. Commun., pages 257-266. Amer. Math. Soc., Providence, RI, 1997. MR1426844

[12] G. N. Watson. A treatise on the theory of Bessel functions. Cambridge Mathematical Library. Cambridge University Press, Cambridge, 1995. Reprint of the second (1944) edition. MR1349110 